\title{
Metadata for describing learning scenarios under the European Higher Education Area paradigm
}

\author{
Ana-Elena Guerrero, Julià Minguillón, Lourdes Guàrdia, and Albert Sangrà \\ Estudis d'Informàtica, Multimèdia i Telecomunicacions \\ Universitat Oberta de Catalunya \\ Rb. Poble nou 156, 08018 Barcelona \\ \{aguerreror, jminguillona, lguardia, asangra\}@uoc.edu
}

\begin{abstract}
In this paper we identify the requirements for creating formal descriptions of learning scenarios designed under the European Higher Education Area paradigm, using competences and learning activities as the basic pieces of the learning process, instead of contents and learning resources, pursuing personalization. Classical arrangements of content based courses are no longer enough to describe all the richness of this new learning process, where user profiles, competences and complex hierarchical itineraries need to be properly combined. We study the intersection with the current IMS Learning Design specification and the additional metadata required for describing such learning scenarios. This new approach involves the use of case based learning and collaborative learning in order to acquire and develop competences, following adaptive learning paths in two structured levels.
\end{abstract}

Keywords: personalization, case based learning, competences, learning design, metadata, ontologies.

\section{Introduction}

The adoption of the European Higher Education Area (EHEA) model, also known as the Bologna process, has changed completely the way institutions need to face educating people, which are now considered to be lifelong learners since the very first moment they start higher education, shifting from contentbased courses to competence development. The design of a competence-based curriculum demands to rethink and redesign programs and courses and stimulates the application of social-constructivist pedagogical approaches that are learner and community-centered. Personalization becomes a useful tool for providing learners with adaptive learning paths according to their specific needs and particularities. With the creation of the new European Higher Education Area, distance and open education is changing the followed approaches until now. As the UNESCO has enunciated, "Universities are important stakeholders in lifelong learning. Their role could evolve, and the link between the learner and the university could become a lifelong link, both to constantly disseminate 
the knowledge and to develop the networks and communities. E-learning should be encouraged and trained to acquire and further develop their e-competences". E-learning courses should guarantee high quality standards, achieving an equal acceptance of skills as those acquired via classical learning, in order to be attractive for professionals. Learners become the center of any educational action and, in the case of blended or virtual learning environments, it is very important to provide them with the appropriate guidance (tutoring, counseling, personalized feedback, etc.) avoiding the classical isolation that learners in distance education suffer from. Furthermore, the new EHEA pushes learners towards a lifelong learning scenario, where education is needed through all of life. Competences are not just "chunks" of information that can be assimilated easily, but must be continuously acquired, developed and updated by means of well designed learning paths, during long periods of time. Personalization is therefore a key factor to help learners to define their starting point in any educational experience, and the paths they need to follow to achieve the desired competence level. Learning paths must be defined taking into account the user profile, but also the hierarchical structure of degrees, courses, subjects, etc., and personalization has a different meaning according to the context where is employed. Nevertheless, learning environments are not prepared yet for describing the learning process based on competences including personalization issues. Furthermore, most of the current e-learning standards and recommendations focus on content description and packaging, and there is only one specification available for describing the learning process (IMS Learning Design, [12]), but it is very limited for providing complete and flexible descriptions for all the elements interacting in a virtual learning scenario taking into account the new requirements of the EHEA paradigm, where activities, resources and learner profiles have to be strongly related with ICT competences for acquiring and developing them in such a learning scenario. As stated in [15], learning designs appear from new organizational designs such as the EHEA, and personalization issues cannot be properly addressed unless standard inter-operability is assured among the elements of the learning process [1]. This paper is organized as follows: Section 2 briefly describes the Universitat Oberta de Catalunya (UOC) experience and the virtual e-learning environment used for supporting lifelong learning and the official masters and degrees. We also describe a pilot educational experience that has been designed following the new requirements imposed by the EHEA paradigm. Section 3 describes the learning scenarios created in this educational experience and the relationship with the current e-learning standards and specifications for describing them. Finally, the discussion of the work presented in this paper, the open issues and the current and future research lines are summarized in Section 4.

\section{Developing competences in a virtual learning scenario}

The Universitat Oberta de Catalunya ${ }^{1}$ (in English Open University of Catalonia) is an institution which has emerged from the knowledge society. The mission is

\footnotetext{
${ }^{1}$ http://www.uoc.edu
} 
to provide people with training throughout their lives. The university's principal aim is to ensure that each student satisfies his/her learning needs in a virtual environment, gaining the maximum benefit from their own efforts. To this end, it offers intensive use of information and communications technologies (ICT), thereby enabling us to overcome the barriers imposed by time and space for offering an educational model based on personalized attention for each individual student. Students, professors and administrators interact and co-operate on our Virtual Campus, constituting a university community which uses the Internet to create, structure, share and disseminate knowledge. Within the UOC virtual campus, each subject has a virtual classroom for teaching and learning process and they are the virtual meeting point for learning activities. Among many other possibilities, the university offers several official degrees and several masters which have been partially or totally adapted to the directives given by the EHEA. One of them is the International Master on Education and ICTs. This master offers several specializations and is oriented towards lifelong learning students who want to improve their knowledge and professional competences in designing, creating and managing different learning scenarios. Within the EHEA adaptation process, all the official degrees and also the offered masters are redesigning and shifting their content-based courses towards learning activities, focusing in the main competences that students have to acquire in order to be competent professionals and/or researchers, depending on the topic. The International Master in Education and ICTs was one of the first courses adapted to the Bologna process, and all the requirements for describing competences, creating adaptive learning paths, personalization issues, using different learning strategies and methodologies and so have arisen from its adaptation.

\subsection{From learning contents to learning activities}

As mentioned before, master degrees are designed as a sequence of subjects which allow learners to create their own learning paths. Learners acquire and develop competences with the aim of achieving the appropriate level for facing real problems in the professional field. The main point is that within each subject learning scenarios are created for facilitating the learning process through the use of a virtual learning environment. Learning resources, activities and a teaching plan (which defines all the details of the learning process) are designed for acquiring the main and specific competences according to the Bologna process for each professional profile. The teaching plan becomes a first step towards a formal description of all the elements involved in the learning process: leaner profiles, competences, activities and learning resources. At the same time, subjects are arranged in semesters and they must be taken according to a higher level plan, taking into account that several competences can be developed in parallel and/or in consecutive subjects. In this sense, learners create their own learning paths, developing competences (both transversal and specific) with the aim of achieving the appropriate level for facing real problems in the professional field or as the first step towards the consecution of a Ph.D. degree, according to their learning goals. Some of these competences are developed across several subjects 
(transversal competences and a few specific ones), while others (the rest of the specific ones) are developed in a single specialization subject. The same competence can be developed at different levels and in different contexts. Therefore, it is important to ensure that the learning path followed by the learner covers all the required competences and the learning goals. This flexibility can be seen as a first step towards a personalized learning process through adaptive formative itineraries, both inter-subject and intra-subject, following a hierarchical structure of two levels. At the bottom level, each subject is thought as a small learning scenario where several learning situations are presented to learners. These situations try to reproduce real life scenarios which involve taking decisions, planning several activities and executing them using the appropriate methodologies, that is, applying real practical knowledge in a professional context. Learners must demonstrate the already acquired competences, by using and developing them, and then incorporating new ones. At the top level, subjects are arranged according to the educational system structure (academic semesters), which is not always coherent with the concept of continuous competence development, as there is not a direct relationship between competences and subjects.

\subsection{Personalization issues}

Personalization is a key aspect for improving user experience and increasing satisfaction, involving him or her into the activity being performed, pursuing fidelity [13]. In other business areas, such as e-commerce, personalization pursues helping users to feel a real personalized service even when buying through an online store. The system remembers all user personal particularities and guides him or her to find the most appropriate resources (in a wide sense) according to his or her needs, with additional recommendations, as Amazon does, for example. Of course, personalization pursues establishing a strong relationship between the customer and the store, in a win-to-win situation. In the particular case of virtual learning environments, personalization has different goals, although not very different from those defined previously: increasing fidelity is a synonym for reducing dropout, the main problem of distance education. As stated in [13], using ideas from the e-commerce model in other fields such as e-learning can be very useful for redefining and improving processes involving customers (learners) and providers (educational institutions). Personalization is crucial under the new EHEA paradigm. Following [2], a meaningful learning is produced when learners are able to connect their knowledge with the previous one they have. That means students can put into practice their competences in each case of study and learn new ones that complement and reinforce their professional skills. On the other hand, developing transversal competences usually involves collaborating with other learners in the same learning scenario, so an interpersonal development is converted in an intrapersonal development by means of social interaction along learning process. Learners have to share their knowledge, skills, points of view and learning resources while they are working in activities in the same virtual learning scenario. Competence development implies designing learning activities which promote using, acquiring and putting 
into practice other competences, which overlap and are semantically related to each other. Competences are usually defined by long textual sentences (i.e. "Developing technological and educational designs related to virtual teaching and learning environments") which makes impossible obtaining formal descriptions unless they are decomposed in smaller pieces, namely learning activities. Personalization is no longer just adapting the contents and/or the syllabus for each student; personalization must be designed using competences as the main element of the learning process. Personalization in virtual learning scenarios can be described according to two dimensions (which are not completely orthogonal): the elements which are taken into account for designing and executing learning paths, namely maps of competences, subjects arranged in learning paths, and learning activities within a subject; and the analysis level used for describing the possible actions [14], namely lifelong learning (long term), academic semester (med term) and user sessions (short term). Table 1 summarizes the intended uses and implications of the combination of both dimensions.

Table 1. Intended uses of personalization in virtual learning environments.

\begin{tabular}{|c|c|c|c|}
\hline & Creating the map & Creating the path & Supervising the path \\
\hline Short Term & - & - & Improving interaction \\
\hline Med Term & Subject planning & Adaptive paths & Learning activities \\
\hline Long Term & Curricula planning & Progressive degrees & Fighting dropout \\
\hline
\end{tabular}

Therefore, designing any long term educational action composed by several subjects that must be combined in order to acquire and develop competences taking into account personalization issues creates new requirements for its formal description that are not available through the use of the current e-learning standards and specifications. It is necessary to evolve from content oriented standards (LOM) to activity based ones (IMS LD) and then towards higher level specifications that allow the description of hierarchical structures such as those required for personalization issues using competences.

\subsection{Towards the Bologna process}

According to the Bologna process, official degrees and master studies have to evolve from content to activities for working the acquisition of general and specific competences. In the case of the International Master on Education and ICTs, it is structured in two academic years (four academic semesters) with 45 ECTS credits, equating to 675 hours of work by the student. Each semester, students enroll in one or more subjects with the aim of acquiring competences oriented to a professional area related with education and ICT, according to their profiles and preferences. All the learning process is supported by the virtual learning environment, which provides students with the teaching plan and 
all the learning resources, following the new EHEA paradigm but using the classical semester-subject structure. The educational proposal includes four types of subjects, with different educational focus depending on the professional skills to be developed: Initial, Basic, Specialization and Application subjects. While the initial and the different basic subjects focus on basic e-learning subjects for the development of a quality professional level of practice, the specialization and application subjects focus on acquiring applied knowledge and consolidating the professional skills that have been developed throughout the master. Students can select and arrange these subjects according to their preferences, but there are several requisites that must be accomplished, especially if one or more competences are developed through one or more subjects. The variety of user profiles and interests creates several possible paths, although most students follow the recommendations given by the teachers and tutors. This creates a complex map of competences and learning paths which needs to be formally described in order to provide learners with recommendations for choosing according to their profile. Figure 1 shows an example of this duality of competence development and subject groups, where one or more subjects are used for developing one or more competences, and each subject uses different methodologies (Case Based Learning or Workgroups).

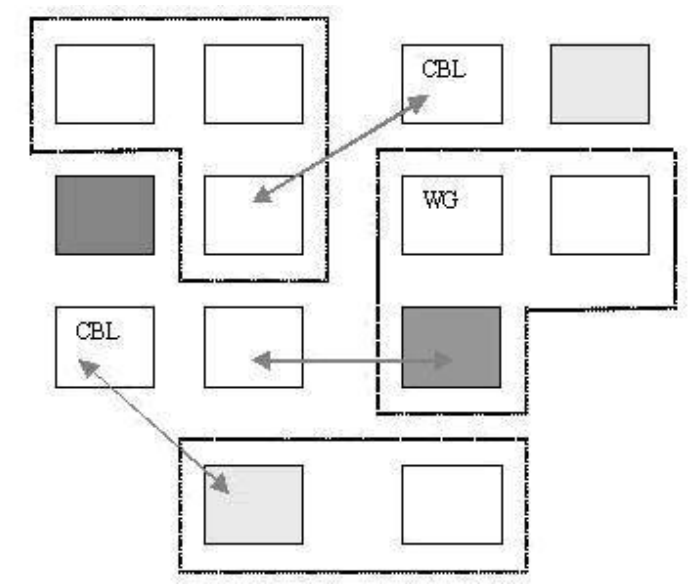

Fig. 1. Examples of learning paths and subject grouping for acquiring and developing competences.

\section{Metadata for describing learning situations and learning scenarios}

Each subject in the official master is designed as one or more learning situations trying to reproduce real professional situations where experts in one field need 
to apply practical knowledge for solving a problem, in a virtual learning environment. This methodology, which tries to ensure a high quality of the learning process, takes into account all the elements in the field of actuation and it reproduces them in terms of learning activities; these activities are designed with all the learning goals in mind, in a hierarchical structure, as follows:

Educational offers

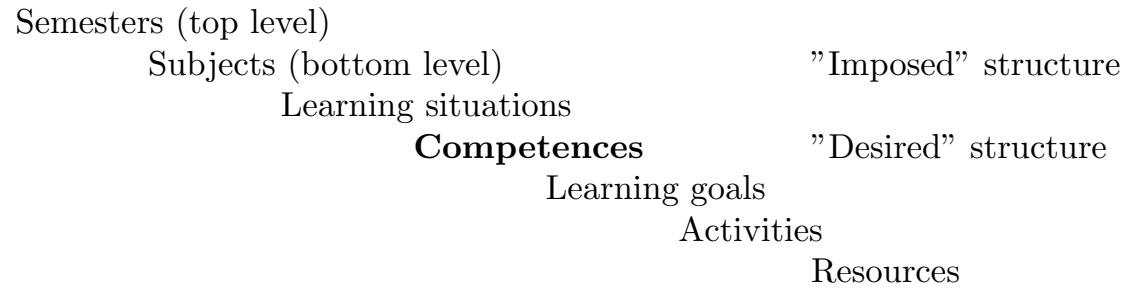

Resources

This structure is currently partially supported by the teaching plan, a document with the description of the subject, which is human-readable, but non machine-readable. Each teaching plan has been designed in basis of three premises:

1. a sound formulation of competences and learning goals;

2. the design of learning activities which are diversified and coherent with the competences to be developed;

3. the design of evaluation activities which prioritize the gathering of evidences that the proposed competences have been properly acquired.

Among other methodological tools, and for the purpose of our research, we define case-based learning (CBL) as an instructional strategy that uses case study as a resource and the case method as the learning scenario description where learners and instructor interact [7]. Most professors using case study describe it as a descriptive document, delivered as a narrative that is based on a real situation or event. The case tries to facilitate a balanced relationship between the multidimensional representation of the context, its participants and the reality of the situation. The concept of case in itself has an internal structure which is independent of the final activities and resources used in its implementation, and this fact can be used to generate different case studies from a subset of case patterns and a collection of learning resources, following an instructional design approach $[7,19]$. Therefore, at the bottom level, we need formal representations for case-based learning scenarios, which involve all the elements in the learning process (learners, activities, competences, resources, etc.), and all the relationships between these elements. Our goal is to provide a mechanism for learning path design according to learner preferences and already acquired competences, learning goals and directions given by instructional designers and teachers. In the particular case of the IMS LD specification, it is necessary to adapt the particular needs of the virtual learning scenario to the specifications 
available following the directions given in [7], where competences are used to describe goals, prerequisites and outcomes of the learning activities. In this sense, there is a lack of standards for describing competences at a rich semantic level, because IMS RDCEO, which is mentioned by IMS LD as a possible competency standard for describing objectives, is not enough to represent the hierarchical structure and all the relationships identified previously, as also stated in [18], where a similar approach is described.

\subsection{Current specifications and standards}

In fact, new standards and specifications such as IMS LD are more oriented towards describing learning scenarios than just contents. The IMS LD specification tries to describe all the aspects and the elements more related to the learning process in itself, such as sequencing or role playing, that is, the second level of description as aforementioned. It seems clear that all this information cannot be stored in the learning objects, but in a higher semantic level. Although the IMS-LD specification may seem too complex for practical applications, its flexibility and multilevel description capabilities allow the specification of any learning process ranking from simple educational itineraries to complex learning processes including personalization and collaborative working capabilities. Nevertheless, both content description standards (such as LOM [9], for example) and learning process description specifications (such as IMS-LD) lack from a formal description for the concept of competence. In [8] a proposal for formal description of required and acquired competences following the most well known taxonomies $[3,4,6]$ is presented, and the use of ontologies for standard integration and extension is discussed, following the approach described in [19]. Other works about competence design and taxonomies in the same direction have been also considered $[16,21]$.

IMS Learning Design In IMS-LD, "Learning-objectives" within an "Activity" is precisely the place to describe competences, but using a more textual approach. Each learning objective is described using, at least, two basic fields, a text based description and a type, which can be one (and only one) of the following: skill, knowledge, insight, attitude, competency and other. Therefore, any extension to include a more comprehensive description of competences should be included here, using the proposal presented in this paper. IMS LD will probably become a standard for defining complex learning processes, including personalization issues. Therefore, it would be interesting to study how to include our proposal in the IMS LD standard taking also into account not only competences but also activities and roles in a personalized learning process. Nevertheless, personalization capabilities of the current LD specification are clearly insufficient for describing the complex requirements of Table 1.

Other e-learning related standards Although LD can be used for describing the learning scenarios needed by each subject, the description of the elements 
of any e-learning process and all the interactions between such elements is not yet a simple question. Two basic levels of description can be identified: the first level, pointed towards content management through the use of learning objects, describes the aspects directly related to the educational content. The second level describes the interactions between such learning objects and the users within the framework defined by the learning process. This separation is needed to ensure reutilization of learning resources in different contexts. For the first level, the LOM standard [9] defines a structure for interoperable descriptions of learning objects. In this case, a learning object is defined as any entity, digital or nondigital, that may be used for learning, education or training. Notice that we do not use the classical definition of learning object from Wiley [20] because it does not include non-digital resources, which are still heavily used at the UOC virtual campus. Nevertheless, although there are several other definitions for learning objects, all of them coincide in a single desired behaviour: reusability. Metadata for a learning object describes relevant characteristics of such learning object to which applies, pursuing reusability. For the second level, we need additional standards and specifications for describing the learning profiles, the competence maps, and the learning process itself beyond LD. As stated in [5], both IEEE Personal and Private Information (PAPI) [10] and IMS Learner Information Package (LIP) [11] overlap in describing user profiles. On the one hand, the main interest of IEEE PAPI is to supply the minimum set with information that can allow follow-up of the student performance during its study. On the other hand, IMS LIP offers richer structures and it takes into the main characteristics of the user, like goals and interests (not covered to IEEE PAPI), and it also describes the characteristics of the student with views to the personalization contents. It is extensible, provides best practice guides and that makes it easier to use and of reading. In any case, it is possible to pass a user profile to IEEE PAPI from IMS LIP. Finally, regarding system architecture issues, the virtual learning scenario should be able to incorporate new services and functionalities when required. Web 2.0 tools, collaborative working and other social software tools will be usual learning scenarios in a near future. OKI [17] includes an Open Service Interface Definition enabling a decentralized system architecture, where services and functionalities are plugged when needed. This is directly related to the concept of teaching plan, where all of these tools need to be described in order to build the virtual classroom.

\subsection{Interoperability issues}

Following [1], it is necessary to establish the models used to describe the learning scenario, namely Domain, User, Context, Instruction and Adaptation. Each model defines one or more elements of the learning process, which are related each other but current standards do not reflect these relationships. Table 2 shows the current standards and specifications that might be used to describe the proposed virtual learning environment with personalization and competence based instructional design capabilities. "P" means that the current standard or specification has a relationship with the concept we want to represent, but only par- 
tially. On the other hand, "C" means that the current standard or specification is enough to fully describe the proposed element.

Table 2. Current standards and specifications for system requirements $(\mathrm{P}=$ partial, $\mathrm{C}=$ Complete).

\begin{tabular}{|l|c|c|c|c|c|}
\hline & LOM & LD & RDCEO & PAPI/LIP & OKI \\
\hline Competence map & & & $\mathrm{C}$ & & \\
\hline Competence profile & & $\mathrm{P}$ & $\mathrm{P}$ & & \\
\hline Competence itineraries & $\mathrm{P}$ & $\mathrm{P}$ & & & \\
\hline Teaching plans & $\mathrm{P}$ & $\mathrm{P}$ & $\mathrm{P}$ & $\mathrm{P}$ & $\mathrm{P}$ \\
\hline Learning situations & $\mathrm{P}$ & $\mathrm{P}$ & & & $\mathrm{P}$ \\
\hline Learning activities & $\mathrm{P}$ & $\mathrm{C}$ & & & $\mathrm{P}$ \\
\hline Learning resources & $\mathrm{C}$ & & & & $\mathrm{P}$ \\
\hline
\end{tabular}

Taking into account the limitations and gaps between this set of standards and specifications is also necessary to create an ontology (or several) for ensuring that all elements involved in a competence based design process are covered. As described in [19], it is possible to use an ontology for describing not only standards, but also the relationships that occur between the elements that take part of such standards, which cannot be part of the learning object instances, thus providing coherence to metadata instances and referring to the appropriate domains. As shown in Table 2, there are main gaps in the competence related concepts which need to be solved for creating learning paths attending personalization issues in a learning scenario based on competences. Ontologies will allow us to add extensions and new rules for covering the disadvantages of the current standards, such as formal definitions for competences or competence maps.

\section{Discussion}

Virtual learning environments are becoming true learning scenarios for both blended and pure virtual distance education. Classical learning content management systems will become obsolete if they just provide learners with contents and syllabus. The new EHEA paradigm, which bases the design of any educational offer in terms of competence acquisition and development, promotes personalization as a way to ensure a proper development for each learner, taking into account his or her particularities, preferences, the already acquired competences and the desired learning goals. Activities for developing competences, and not contents, are the basic pieces for designing educational experiences. This means that classical e-learning standards and specifications need to be rethought in order to incorporate this new vision. Furthermore, personalization means different things depending on the elements taken into account and the level of application, and the complexity of all the possibilities is beyond the capacity of the 
current standards. The IMS LD specification is the first step towards a complete description of the elements in the learning process, although it is still far from providing complete descriptions with the required level of detail, including adaptive learning paths for competence development. It needs to be used in combination with other e-learning standards and specifications, and semantic interoperability is not always ensured and, in fact, very limited. Therefore, the use of ontologies for connecting all the elements present in the learning process is a possible way for solving this interoperability gaps and bridging the different standards and specifications. The UOC virtual campus is undergoing a major revision (both in technological and methodological aspects) with the inclusion of new web 2.0 tools for teaching and learning, personalization issues and adopting a web services based architecture using OKI. The International Master on Education and ICTs will be used as a pilot experience for providing learners with adaptive learning paths and a complete curriculum based on competence evaluation, acquisition and development. The concept of teaching plan is also under development as it is clear that it cannot be only a sequence of learning activities involving the use of learning resources, but it has to reflect the higher level competence maps and personalization issues through the use of user profiles. Current and future research lines in this subject include the creation of pilot subjects including both case based learning and workgroups, using LD as the starting point for the formal definitions. As several LD based courses will be available, a higher level definition taking into account the competence map will be needed to provide the adaptive learning paths according to user profile. The use of ontologies for creating relationships between the elements described by different e-learning standards and recommendations is also an interesting issue which needs to be deeply developed yet.

\section{Acknowledgements}

This paper has been partially supported by a Spanish government grant under the project PERSONAL (TIN2006-15107-C02-01). 